\title{
Responsible conduct in animal research
}

Laboratory animals play a crucial role in biomedical research - indeed many advances now incorporated into human health care would have been impossible without them. Informed and well-trained scientists have the privilege, but not the automatic right, to use animals as experimental subjects. This privilege must not be abused.

J. Clin. Invest. 112:1456 (2003). doi:10.1172/JCI200320394.

tress. We must not take the responsibilities of animal care lightly.

As researchers ourselves, we understand that the scientific process can entail frustrations and disappointments. Experiments may fail for a number of reasons - technical complications or shortages of time, supplies, or students. But once a manuscript is published, the data held within can be freely used by other researchers to extend their

In the current issue of the JCI, we are issuing a retraction of a paper on the role of inducible costimulator (ICOS) in rheumatoid arthritis first published in March 2003. The article shows that in the absence of ICOS, mice normally susceptible to collagen-induced arthritis were completely protected from the disease. These results identified ICOS as a potential target for therapeutic intervention in rheumatoid arthritis.

While the paper was well received during peer review and may still contain legitimate ideas and findings relevant for the treatment of arthritis, the Editors of the JCI became aware soon after publication that there was a problem. As part of the manuscript submission process, we require authors whose work contains data procured from animal or human subjects to explicitly declare that the research was undertaken under the auspices of a protocol approved by an institutional review board (IRB) or institutional animal care and usage committee (IACUC). In this case, a declaration of this nature was made in the submitted and published manuscript, but it was not entirely accurate.

For both ethical and scientific reasons, all individuals whose hypotheses require validation through the use of laboratory animals must take the time to learn how to properly and ethically treat research subjects. Authors' institutions must carefully review experimental protocols, and experiments should proceed only after approval has been granted. While trials may go awry or unintended reactions may occur during the course of experimentation, working closely with IACUCs is imperative. It is the responsibility of researchers and IACUCs to determine the best course of experimental procedures, especially when the chosen experiments cause animals to feel any dis- own observations and, we hope, to affect treatment of human patients. For this reason, the onus is on the researcher to present a manuscript that can become part of the public record.

Our contribution to the scientific public record is built on a foundation of trust and integrity. The community has to be able to trust that the results we report from others are valid. Research journals play a vital role in the advancement of science through certification and dissemination of findings from authors to readers. In essence, journals place a stamp of credibility on data published within their pages, so it is essential that the findings reported therein are not only scientifically legitimate but also ethically sound.

The assumption that each author meets our scientific and ethical standards during the review process has allowed findings published in our journal to ultimately be translated to clinical therapies. But this trust will only endure if our authors are devoted to exemplifying the values associated with ethical scientific conduct. We have come to rely on our authors' integrity as our readers rely on ours; we cannot police each author. In this case, we proceeded with peer review as the authors explicitly declared within their manuscript that their institution had approved the experiments presented.

We realize that IACUC policies and procedures can be complex, and it is our understanding that the authors are working closely with their IACUC to review the approval process in order to determine where the miscommunication occurred. However, the authors of the paper have agreed that at this time, under the policies of the JCI, which clearly state that all experiments must be formally approved by an IRB or IACUC, publication of the article cannot stand.

Ushma Savla, Ph.D.

Executive Editor 